\title{
AUTHOR INDEX VOLUME 10 (2002)
}

Ashkenazy, Y. \& Horwitz, L.P., The radiative kicked oscillator: a stochastic web or chaotic attractor?

10 (2002) 353-371

Balasco, M., Heinicke, J., Koch, U., Martinelli, G. \& Telesca, L., Fractal analysis of the hourly time variability in self-potential and flow variations concomitantly measured in the Soos Nature Park (Czech Republic)

Barraclough, D. see De Santis, A.

Castrejón García, R., see Castrejón Pita, J.R.

Castrejón Pita, J.R., Sarmiento Galán, A. \& Castrejón García, R.

Chacón, O., see González, V.A.

Choi, J.S., see Kim, K.

Consolini, G., Self-organized criticality: a new paradigm for the magnetotail dynamics

Croft, J.C., see Jacka, S.D.

Cuomo, V., see Telesca, L.

Dalla, L., Bivariate fractal interpolation functions on grids

De Santis, A., Barraclough, D. \& Tozzi, R.

Dekking, F.M. \& Van der Wal, P., The boundary of the attractor of a recurrent iterated function system

Devaney, R.L. \& Rocha, M.M., Geometry of the antennas in the Mandelbrot set

Ehrenberger, K., see Shimizu, Y.

Einstein, A.J., Wu, H.-S. \& Gil, J., Detrended fluctuation analysis of chromatin texture for diagnosis in breast cytology

Fanchiotti, H., García Canal, C.A. \& Martínez, N., Critical analysis of electronic simulation of financial market fluctuations

Furcolo, P., see Veneziano, D.

Furcolo, P., see Veneziano, D.

Gaonac'h, H., see Harvey, D.C.

García Canal, C.A., see Fanchiotti, H.

Gil, J., see Einstein, A.J.

Goldburg, W., Intermittency in turbulent flows

González, V.A., Chacón, O., Hinojosa, M. \& Guerrero, C., Statistical assessment of self-affine methods applied to short profiles

Greiner, M. \& Jouault, B., An experimentalist's view of discrete and continuous cascade models in fully developed turbulence

Guerrero, C., see González, V.A.

Hambly, B.M. \& Kumagai, T., Asymptotics for the spectral and walk dimension as fractals approach Euclidean space

Harvey, D.C., Gaonac'h, H., Lovejoy, S., Stix, J. \& Schertzer, D., Multifractal characterization of remotely sensed volcanic features: a case study from Kilauea volcano, Hawaii

Heinicke, J., see Balasco, M.

Henry, B.I., see Kim, K.

Hinojosa, M., see González, V.A.

Horwitz, L.P., see Ashkenazy, Y.

Huillet, T., On the waiting time paradox and related topics

10 (2002) 463-472

10 (2002) 297-303

10 (2002) 429-434

10 (2002) 429-434

10 (2002) 373-386

10 (2002) 229-233

10 (2002) 275-283

10 (2002) 199-208

10 (2002) 67-76

10 (2002) 53-58

10 (2002) 297-303

10 (2002) 77-89

10 (2002) 39-46

10 (2002) 103-116

$10(2002) 19-25$

10 (2002) 473-479

10 (2002) 147-156

10 (2002) 209-222

10 (2002) 265-274

10 (2002) 473-479

10 (2002) 19-25

10 (2002) 131-133

10 (2002) 373-386

10 (2002) 321-327

10 (2002) 373-386

10 (2002) 403-412

10 (2002) 265-274

10 (2002) 463-472

10 (2002) 229-233

10 (2002) 373-386

10 (2002) 353-371

10 (2002) 173-188 
Iacobellis, V., see Veneziano, D.

10 (2002) 209-222

Jacka, S.D. \& Croft, J.C., The Hausdorff dimension of some snowflake-like recursive constructions

Jouault, B., see Greiner, M.

Kim, G.H., see Kim, K.

Kim, K., Kim, G.H., Lee, J.R., Choi, J.S., Kong, Y.S., Henry, B.I., Yum, M.K. \&

Odagaki, T., Multifractal measures in fractional iterative maps

Koch, U., see Balasco, M.

Kong, Y.S., see Kim, K.

Kruhl, J.H., see Suteanu, C.

Kumagai, T., see Hambly, B.M.

Kumagai, Y., Fractal structure of financial high frequency data

Lantsman, Y., Major, J.A. \& Mangano, J.J., On the multifractal distribution of insured property

Lapenna, V., see Telesca, L.

Lauren, M.K. \& Stephen, R.T., Fractals and combat modeling: using MANA to explore the role of entropy in complexity science

Lee, J.R., see Kim, K.

Liaw, S.-S., Find the Mandelbrot-like sets in any mapping

Lovejoy, S., see Harvey, D.C.

Lovejoy, S., see Sachs, D.

Luo, J., A note on a self-similar tiling generated by the minimal pisot number

Luo, J., Rao, H. \& Tan, B., Topological structure of self-similar sets

L'vov, V.S., see Pierotti, D.

Macchiato, M., see Telesca, L.

Major, J.A., see Lantsman, Y.

Mangano, J.J., see Lantsman, Y.

Martínez, N., see Fanchiotti, H.

Martinelli, G., see Balasco, M.

Moiseev, S.S. \& Pungin, V.G., Analysis of nonlinear development of helical vortex instability

Monte, E., Roca, J. \& Vilardell, L., On the self-similar distribution of the emergency ward arrivals time series

Montemurro, M.A. \& Pury, P.A., Long-range fractal correlations in literary corpora

Nicolis, C., see Schmitt, F.

Obregón, N., Puente, C.E. \& Sivakumar, B., Modeling high-resolution rain rates via a deterministic fractal-multifractal approach

Obregón, N., see Puente, C.E.

Ochiai, F., see Takehara, T.

Odagaki, T., see Kim, K.

Oldershaw, R.L., Mass estimates for galactic dark matter objects as a test of a fractal cosmological paradigm

Pierotti, D., L'vov, V.S., Pomyalov, A. \& Procaccia, I., Birth of anomalous scaling in a model of hydrodynamic turbulence with a tunable parameter

Pommois, P., Veltri, P. \& Zimbardo, G., Diffusive and anomalous magnetic field lines transport in anisotropic turbulence

Pomyalov, A. see Pierotti, D.

Procaccia, I., see Pierotti, D.

Puente, C.E., see Obregón, N.

Puente, C.E., Obregón, N. \& Sivakumar, B., Chaos and stochasticity in deterministically generated multifractal measures

Pungin, V.G., see Moiseev, S.S.

Pury, P.A., see Montemurro, M.A.

Rao, H., see Luo, J.

Richards, G.R., Fractality in a macroeconomic model: nonlinear oscillation around a long-term equilibrium

Roca, J., see Monte, E.

10 (2002) 199-208

10 (2002) 321-327

10 (2002) 229-233

10 (2002) 229-233

10 (2002) 463-472

10 (2002) 229-233

10 (2002) 435-449

10 (2002) 403-412

10 (2002) 13-18

10 (2002) 305-311

10 (2002) 67-76

10 (2002) 481-489

10 (2002) 229-233

10 (2002) 137-146

10 (2002) 265-274

10 (2002) 253-264

10 (2002) 335-339

10 (2002) 223-227

10 (2002) 291-296

10 (2002) 67-76

10 (2002) 305-311

10 (2002) 305-311

10 (2002) 473-479

10 (2002) 463-472

10 (2002) 395-402

10 (2002) 413-427

10 (2002) 451-461

10 (2002) 285-290

10 (2002) 387-394

10 (2002) 91-102

10 (2002) 47-52

10 (2002) 229-233

10 (2002) 27-38

10 (2002) 291-296

10 (2002) 313-319

10 (2002) 291-296

10 (2002) 291-296

10 (2002) 387-394

10 (2002) 91-102

10 (2002) 395-402

10 (2002) 451-461

10 (2002) 223-227

10 (2002) 235-251

10 (2002) 413-427 
Rocha, M.M., see Devaney, R.L.

Sachs, D., Lovejoy, S. \& Schertzer, D., The multifractal scaling of cloud radiances from $1 \mathrm{~m}$ to $1 \mathrm{~km}$

Sarmiento Galán, A., see Castrejón Pita, J.R.

Schertzer, D., see Harvey, D.C.

Schertzer, D., see Sachs, D.

Schmitt, F. \& Nicolis, C., Scaling of return times for a high-resolution rainfall time series

Shimizu, Y., Thurner, S. \& Ehrenberger, K., Multifractal spectra as a measure of complexity in human posture

Simon, K. \& Solomyak, B., On the dimension of self-similar sets

Sivakumar, B., see Obregón, N.

Sivakumar, B., see Puente, C.E.

Solomyak, B., see Simon, K.

Stephen, R.T., see Lauren, M.K.

Stix, J., see Harvey, D.C.

Suteanu, C. \& Kruhl, J.H., Investigation of heterogeneous scaling intervals exemplified by sutured quartz grain boundaries

Suzuki, N., see Takehara, T.

Tachimori, Y. \& Tahara, T., Clinical diagnoses following Zipf's law

Tahara, T., see Tachimori, Y.

Takehara, T. Ochiai, F. \& Suzuki, N., Fractals in emotional facial expression recognition

Tan, B., see Luo, J.

Tchiguirinskaia, I., Scale invariance and stratification: the unified multifractal model of hydraulic conductivity

Telesca, L., see Balasco, M.

Telesca, L., Cuomo, V., Lapenna, V. \& Macchiato, M., Fractal characterization of the temporal distribution of aftershocks associated with the $1994 \mathrm{M}_{\mathrm{W}} 6.7$ Northridge earthquake

Thurner, S., see Shimizu, Y.

Tozzi, R. see De Santis, A.

Van der Wal, P., see Dekking, F.M.

Veltri, P., see Pommois, P.

Veneziano, D. \& Furcolo, P., Scaling of multifractal measures under affine transformations

Veneziano, D., Furcolo, P. \& Iacobellis, V., Multifractality of iterated pulse processes with pulse amplitudes generated by a random cascade

Veneziano, D., Iterated random pulse processes and their spectral properties

Veneziano, D., Large deviations of multifractal measures

Vilardell, L., see Monte, E.

Wang, P.-J. \& Wang, R.-Y., A generalized width function of fractal river network for the calculation of hydrologic responses

Wang, R.-Y., see Wang, P.-J.

Wu, H.-S., see Einstein, A.J.

Yavari, A., Generalization of Barenblatt's cohesive fracture theory for fractal cracks

Yum, M.K., see Kim, K.

Zimbardo, G., see Pommois, P.
$10(2002) 39-46$

10 (2002) 253-264

10 (2002) 429-434

10 (2002) 265-274

10 (2002) 253-264

10 (2002) 285-290

10 (2002) 103-116

10 (2002) 59-65

10 (2002) 387-394

10 (2002) 91-102

10 (2002) 59-65

10 (2002) 481-489

10 (2002) 265-274

10 (2002) 435-449

10 (2002) 47-52

10 (2002) 341-351

10 (2002) 341-351

10 (2002) 47-52

10 (2002) 223-227

10 (2002) 329-334

10 (2002) 463-472

10 (2002) 67-76

10 (2002) 103-116

10 (2002) 297-303

10 (2002) 77-89

10 (2002) 313-319

10 (2002) 147-156

10 (2002) 209-222

10 (2002) 1-11

10 (2002) 117-129

10 (2002) 413-427

10 (2002) 157-171

10 (2002) 157-171

10 (2002) 19-25

10 (2002) 189-198

10 (2002) 229-233

10 (2002) 313-319 